OTTO KRESTEN / WIEN

\title{
EINE KONJEKTUR ZU DE CERIMONIIS I 86: INBESTIATON*
}

In jenen Abschnitten am Ende des „Liber primus“ des sogenannten „Zeremonienbuches“ des Kaisers Konstantinos VII. Porphyrogennetos, die entweder ausdrücklich unter dem Namen des Petros Patrikios laufen (I

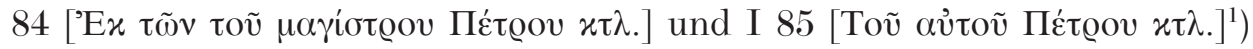
oder die diesem Autor aus der Zeit des Kaisers Iustinianos I. mit guten Gründen zugeschrieben werden (I 86-95 $5^{2}$, befindet sich auch eine Passa-

* Die Anregung zur Abfassung der vorliegenden Miszelle erhielt der Autor durch eine Lektüre der (einstweilen nur im Typoskript vorliegenden) Habilitationsschrift von Cl. SodE, Die Krönungsprotokolle des Petros Patrikios im Zeremonienbuch Konstantins VII. Porphyrogennetos. Jena (Philosophische Fakultät der Friedrich-Schiller-Universität) 2004. - Da die Arbeit von Sode eine auf dem aktuellen Forschungsstand befindliche Bibliographie zu den im folgenden kurz angeschnittenen Fragen (etwa zu dem unter dem Namen des Kaisers Konstantinos VII. Porphyrogennetos laufenden „Zeremonienbuch" oder zu den in De cerimoniis übernommenen Texten des Petros Patrikios und zu den vermutbaren Gründen dieser Übernahme) enthält, kann davon Abstand genommen werden, die Fußnoten der vorliegenden Miszelle mit bibliographischen Angaben zu überlasten, die in aller gewünschten Vollständigkeit bei Sode (bzw. in der kommenden Druckfassung ihrer Habilitationsschrift) nachgelesen werden können (ebenso wird darauf verzichtet, hier die Ansichten des Verfassers zur Genesis des sogenannten „Zeremonienbuches“ und zur vermuteten „Endredaktion“ durch Basileios Parakoimomenos — vgl. dazu etwa O. Kresten, Sprachliche und inhaltliche Beobachtungen zu Kapitel I 96 des sogenannten „Zeremonienbuches“. BZ 93 [2000] 474-489; zu einer anderen Position vgl. M. Featherstone, Preliminary Remarks on the Leipzig Manuscript of $D e$ cerimoniis. BZ 95 [2002] 457-479 — longe lateque zu wiederholen). — Im folgenden (über die Verweise auf Sode hinaus) verwendete Abkürzungen: REISKE (I und II) = Constantini Porphyrogeniti imperatoris De cerimoniis aulae byzantinae libri duo graece et latine e recensione Io. Iac. REISKII cum eiusdem commentariis integris, Bd. I-II (CSHB VII/1 - 2). Bonn 1829-1830; Lips. bzw. Lipsiensis = Leipzig, Universitätsbibliothek, Cod. Rep. I 17 (gr. 28) (mit Verweis auf das entsprechende Folium, gegebenenfalls auch auf die entsprechende Zeile).

${ }^{1}$ Reiske I 386, 24 und 388, 2.

2 Abschnitte über (weitere) Ämterverleihungen, über den Empfang von auswärtigen (,westlichen“ und persischen) Gesandtschaften und „Krönungsprotokolle“ aus der Zeit der Kaiser Leon I. bis Iustinianos I.: vgl. Sode, passim. 
ge (zur Ernennung eines Silentiarios durch den Kaiser), die in der derzeit gedruckten (und im Grunde nur in dieser Form von der Forschung benutzten) Fassung einige lexikologische Probleme aufwirft. Zur „Ernennungsurkunde" (für die in anderen, benachbarten Abschnitten, etwa in Kap. I

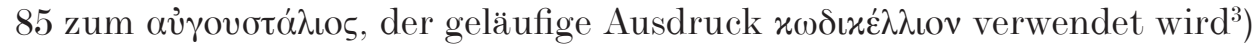

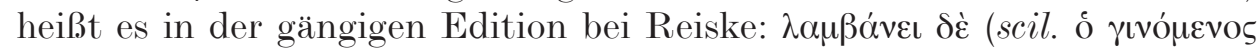

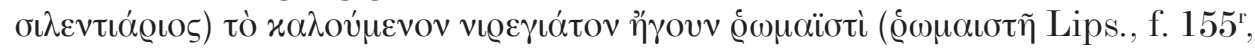

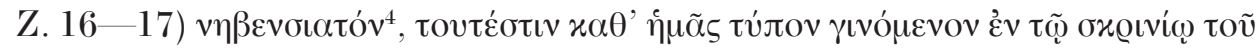

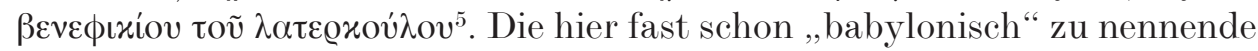
Sprachverwirrung dürfte schon frühzeitig eingesetzt haben, denn auf sie ist wohl eine im Leipziger Codex auf f. 155 (im rechten Freirand) zu lesende, von erster Hand stammende (d. h. aus der direkten Vorlage des Lipsiensis übernommene) Marginalie zurückzuführen, die — pseudogelehrt

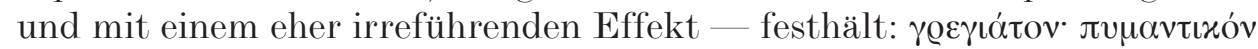

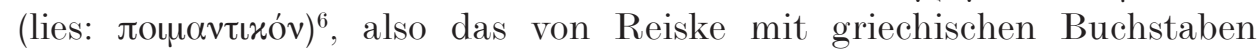

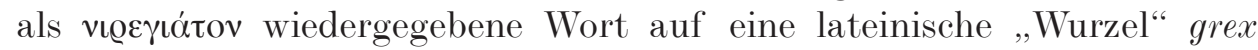
zurückführen möchte. „Pseudogelehrt" ist leider auch das Verdikt, das in diesem Falle über Reiskes kommentierende Erklärungsversuche gefällt werden muß: „Putabat homo, quia grex лoíuv temporis pronuntiabant, gregiatum (nostro scribendi more gretschatum) esse лouavtixòv, pastorium. Non incommode quidem pastorium appellaretur

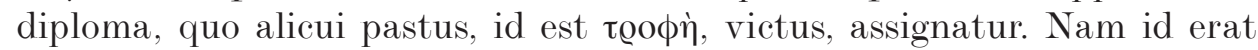
gregatum, aut impensatum, quo alio nomine idem significatur. Verum tamen gregatum hoc non est, sed est mutilatum initio aggregatum, charta, qua

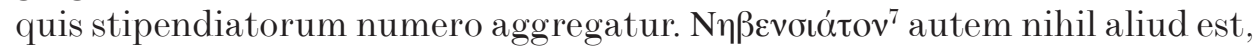
quam deformatum a Graeculis 8 impensatum. ... Erat autem impensatum charta vel diploma, codicillus, quo significabatur rationali largitionum, impensas in exhibentem deinceps faciendas esse, illum porro unum fore eorum, qui pensiones seu stipendia e fisco acciperent ${ }^{\star 9}$.

\footnotetext{
${ }^{3}$ Reiske I 388, 6.

${ }^{4}$ An dem Umstand, daß von den beiden „Schlüsselwörtern“ im Lipsiensis das eine ein Paroxytonon, das andere ein Oxytonon ist, scheint sich bisher noch niemand gestoßen zu haben.

${ }^{5}$ Reiske I 389, 8-11.

${ }^{6}$ Reiske I 389, App. zu Z. 8; s. auch Sode 87, Anm. 8.

${ }^{7}$ Daß hier aus einem Oxytonon des Textes im Kommentar ein Paroxytonon gemacht wird, übergeht Reiske stillschweigend.

${ }^{8}$ Man sieht: Nationale Vorurteile sind ebenso alt wie zäh und langlebig. Reiske befleißigt sich hier einer Ausdrucksweise, die der mittelalterlichen „westlichen“ Propaganda gegen die Byzantiner durchaus geläufig war.

9 Reiske II 371.
} 
Daß angesichts dieser „Ausgangslage“ die weiteren Versuche, die interpretatorische crux der zitierten Passage aus De cer. I 86 zu klären, eher beschränkt waren, liegt auf der Hand ${ }^{10}$. Das $\pi \varrho \tilde{\omega} \tau o v \psi \varepsilon \tilde{v} \delta o \varsigma$, das die richtige Deutung dieser Stelle so schwierig macht, liegt in der unzutreffenden Art und Weise der Wiedergabe des vı@eүı́́ Reiske, die das Faktum mißachtet, daß dieses Wort im Lipsiensis (f. 155 Z. 16) in lateinischen Buchstaben geschrieben ist:

\section{hiREGidioh}

Über die philologischen Konsequenzen hinaus, die mit dieser Beobachtung zu verknüpfen sind, lohnt es auch, das Wort unter Gesichtspunkten der lateinischen Paläographie zu betrachten, auch wenn es natürlich evident ist, daß die Buchstabengestaltung zwischen dem „Autographon“ des Petros Patrikios und der Wiedergabe im Leipziger Codex des „Zeremonienbuches“11 (bei allem Bemühen um ein korrektes „Nachmalen“) einigen (auch ärgeren) Deformationen und Mißverständnissen ausgesetzt war ${ }^{12}$. Zunächst ist es klar, daß die lateinische Schrift, die hier für dieses Wort zur Anwendung kommt, der Uncialis zuzuweisen ist, wie etwa die „Leitbuchstaben" $R$ und $T^{13}$ eindeutig belegen ${ }^{14}$. Darüber hinaus kann es als gesichert

${ }^{10}$ Von der bei Sode 87, Anm. 7-9, zitierten Literatur, die sich, quasi ex officio, mit der Problematik dieser Stelle hätte auseinandersetzen müssen, wagt nur LBG I 330

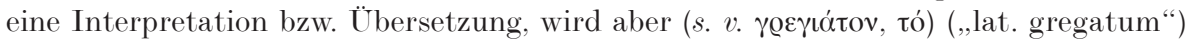
(,eine Urkunde“) bedauerlicherweise Opfer der irreführenden Marginalie auf f. 155 des Lipsiensis.

11 Dessen Entstehungszeit ich nach wie vor am ehesten in die Jahre zwischen 963 und 969 verlegen möchte.

12 Man beachte fürs erste nur die sehr ungeschickte Gestaltung des $N$, die an eine Form erinnert, die der Schreiber des Lipsiensis ansonsten für griechisches Minuskel-Eta gebraucht, wenn es keine Ligatur mit dem folgenden Buchstaben eingeht (vgl. z. B.

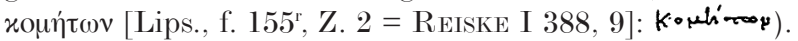

13 Für die in einer Semiuncialis, die in einem „Ausgangscodex“ des 6. Jahrhunderts keinesfalls ausgeschlossen werden dürfte, eindeutig andere Formen zu erwarten wären.

14 En passant: Der Umstand, daß auch in De cer. I 84 (wo die Überschrift noch ausdrücklich auf die Verfasserschaft des Petros Patrikios an dem hier ausgewerteten Text verweist) ein lateinisches Wort - $\lambda \subset \in \boldsymbol{L} \tau \in \mathbf{s}$ - in lateinischen Buchstaben geschrieben wird (Lips., f. 154 ${ }^{\mathrm{v}}, \mathrm{Z} .21$ = Reiske I 387, 16 [wiederum in griechischen Lettern, d. h. von neuem unter irreführender Unterdrückung des paläographischen Befundes; vgl. auch

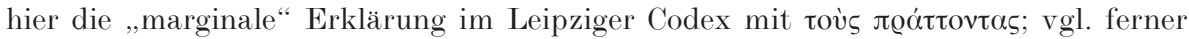
SODE 64f. mit Anm. 5]), und zwar ebenfalls in Uncialis (vgl. etwa die „Leitbuchstaben“ $E, S$ und $T$; s. auch das $N$ in der nämlichen ungeschickten Form, die soeben in Anm. 12 besprochen wurde), könnte als zusätzlicher Beleg für die Autorschaft des Petros Patrikios an dem in De cer. I 86 herangezogenen Werk gewertet werden (und zwar unter der Voraussetzung, daß die Abschnitte De cer. I 84 und De cer. I 86 offensichtlich 
gelten, daß die Worte, die hier im Lipsiensis in De cer. I 86 mit NIR- bzw. mit $v \eta \beta$ - beginnen, nicht zwei verschiedene Begriffe darstellen, sondern ein und denselben (lateinischen) terminus technicus wiedergeben wollen,

auf ein und dieselbe Vorlage zurückgegriffen haben, in der von Fall zu Fall lateinische termini technici innerhalb eines ansonsten griechischen Textes in (relativ sorgfältiger) lateinischer Uncialis geschrieben waren. - Dieses Beobachtung erlaubt nicht nur die Anregung, daß eine sorgfältige durchgehende paläographische Untersuchung der im Lipsiensis (und in den gleichzeitigen, ein und demselben „Muttercodex“ entstammenden

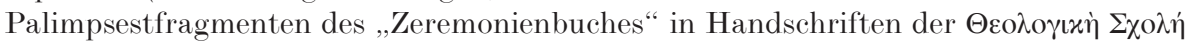
der Insel Chalke und des Athos-Klosters Batopediu: vgl. dazu Sode 28 mit Anm. 3-5) vorhandenen "Lateinworte“ aufgrund möglicher differenzierter lateinischer Buchstabenformen - mögen sie nun der Uncialis, der Semiuncialis oder der jüngeren römischen Kursive entstammen - ein zusätzliches Argument bei der Unterscheidung der jeweiligen (älteren) Quellen darstellen könnte, wie sie dann im 10. Jahrhundert im sogenannten ,Zeremonienbuch“ ausgewertet wurden, sondern ermöglicht darüber hinaus die Vermutung, daß ebendiese Detailstudien zur lateinischen Paläographie auch ein wichtiges Wort bei der Klärung der stemmatischen Zusammenhänge zwischen dem Lipsiensis und den Palimpsesten (so die nämlichen Passagen auch in diesen Fragmenten enthalten sind) mitreden könnten. Und noch eines: SoDE vermutet des öfteren (vgl. etwa die Zusammenfassung auf S. 244), daß so manches an textlichen Unzulänglichkeiten, wie sie sich in De cer. I 84-95 finden, darauf zurückzuführen sein könnte, daß man im 10. Jahrhundert hier ein (eben per se unzulängliches) „Arbeitsexemplar“ des Petros Patrikios („das aus verschiedenen Einzelsammlungen, Heften, Blättern und losen Zetteln sowie einer Vielzahl von Randnotizen bestand") herangezogen hat. Wie sich diese Hypothese mit dem Umstand verträgt, daß die Lateinworte in diesem „Arbeitsexemplar" in ziemlich sauberer Uncialis geschrieben waren, müßte jetzt neu überdacht werden: Für ein „Arbeitsexemplar“ des Petros Patrikios aus der Zeit um 550 würde man an sich Eintragungen in lateinischer Schrift in einer nicht gerade eleganten jüngeren römischen Kursive erwarten, wie sie sich etwa in den „Lateinworten“ oder in den lateinischen Buchstaben mancher Passagen (kopial überlieferter) byzantinischer Kaiserurkunden des ausgehenden 9. und des beginnenden 10. Jahrhunderts oder in einigen Originalen der byzantinischen Kaiserkanzlei aus der zweiten Hälfte des 11. Jahrhunderts und vom Beginn des 12. Jahrhunderts widerspiegelt (vgl. etwa Abb. 1 und 2/II in: Actes du Prôtaton. Édition diplomatique par D. Papachryssanthou [Archives de l'Athos VII]. Album. Paris 1975, oder Abb. 17-20 bei F. Dölger, Facsimiles byzantinischer Kaiserurkunden. München 1931; auf die Anführung von Belegen für das schließlich kaum mehr verstandene Rekognitionswort legimus in byzantinischen Kaiserurkunden bis ins ausgehende 12. Jahrhundert sei hier verzichtet). - Nicht bestritten sei hingegen das (von Sode mit guten Gründen betonte) Faktum, daß gerade die in der vorliegenden Miszelle diskutierte Passage aus De cer. I 86 darauf hindeutet, daß hier mehrere (zunächst interlinear oder marginal angebrachte) „,kommentierende“ Notizen (stufenweise, d. h. in verschiedenen Phasen?) in den Grundtext eingedrungen sein könnten (vgl. dazu auch den Hinweis in der folgenden Anmerkung), ein Vorgang,

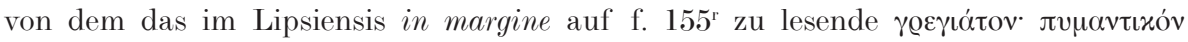
(vgl. oben, Anm. 6) die zeitlich letzte (nicht mehr zur „Ehre“ der Aufnahme in den Haupttext gelangte) Stufe repräsentieren könnte. 
und zwar einmal in lateinischen, dann in griechischen Buchstaben ${ }^{15}$. Für die Buchstaben $I /$ Eta wird man das wohl nicht eigens begründen müssen; für das „Schwanken“ zwischen $R$ und Beta genügt der Hinweis, daß gerade in der lateinischen Uncialis der spätantiken und frühmittelalterlichen Jahrhunderte eine Verwechslung der (durchwegs ähnlich geschriebenen) Buchstaben $R$ und $B$ möglich ist (was etwa für die Semiuncialis oder für die jüngere römische Kursive a limine auszuschließen wäre $)^{16}$.

Freilich - den Beginn eines sinnvollen, auf eine „Ernennungsurkunde“ eines Silentiarios zu beziehenden (lateinischen) Wortes ergibt die Buchstabenabfolge NIR/NIB noch immer nicht; aber auch hier hat der nächste Schritt eines Deutungsversuchs den Weg über Phänomene der lateinischen Uncialis zu gehen, über eine Schrift, in der verlesende Inversionen der Buchstabenfolge $I N$ in ein $N I$ keineswegs nur vereinzelt nachweisbar $\operatorname{sind}^{17}$. Auch diese Annahme scheint zunächst in eine Sackgasse zu führen, da nach den (klassischen) Assimilationsregeln der lateinischen Sprache ein $I N R$ - zu einem $I R R$ - und ein $I N B$ - zu einem $I M B$ - werden müßte. Doch wenn man von der Voraussetzung ausgeht, daß im griechischen Sprachbereich (gerade des 6 . Jahrhunderts und natürlich auch der folgenden Jahrhunderte) lateinisches $V$ praktisch ,gleichwertig“ mit lateinischem $B$ war, so ergibt sich als Beginn des gesuchten lateinisches Begriffes ein $I N V E{ }^{-18}$, d. h. mit

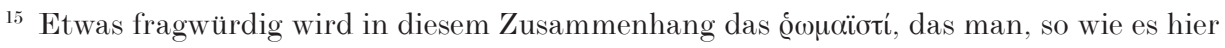
im Text steht, eigentlich nur mit „,in griechischen Buchstaben“ übersetzen könnte, was aber mit dem sonstigen (freilich auch anderen Quellen entstammenden) Sprachgebrauch des „Zeremonienbuches" nicht in Deckungsgleiche zu bringen ist (vgl. etwa De cer. I

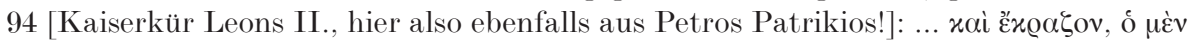

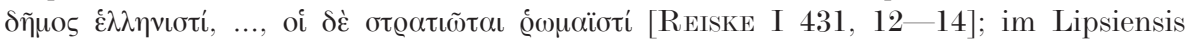
[f. $168^{\mathrm{r}}$ ] an dieser Stelle keine lateinischen Buchstaben verwendet). Die Lösung dieses kleinen „Rätsels“ könnte darin liegen, daß man beim Kopieren des Textes des Petros Patrikios in das "Zeremonienbuch" einfach die Reihenfolge der griechischen und lateinischen Buchstaben vertauscht hat (was ohne weiteres auch darauf zurückzuführen sein könnte, daß die eine „Version“ interlinear oder marginal angebracht war).

${ }^{16}$ Unter dem Gesichtspunkt von möglichen (eine Interpretation erschwerenden) graphischen Verwechslungen und Verlesungen sei schon hier darauf verwiesen, daß auch die lateinische Buchstabenabfolge -EGIA- und das griechische - $\varepsilon v \sigma \iota-$-den Mittelteil ein und desselben (lateinischen) Wortes wiedergeben.

${ }^{17}$ Eine Erklärung, die wieder (vor allem) an die Uncialis gebunden ist, die zwar auch in den verschiedenen Formen der Capitalis möglich wäre, hingegen aber nicht (oder so gut wie nicht) in der Semiuncialis.

18 Als mögliche Vergleichsbeispiele mögen griechisches ı̊v $\beta \varepsilon x \tau o \varsigma$ (für lateinisches invectus,

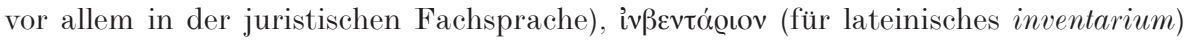
und '̌vßsvtov (für lateinisches inventum) dienen: vgl. LBG I 709 (und die dort angeführten Belege; s. besonders den „Index verborum latinorum“ in: Lexica Iuridica Byzantina ediderunt L. Burgmann-M. Th. Fögen-R. Meijering-B. H. Stolte. Frankfurt/Main 1990 [= Fontes Minores VIII], 429). 


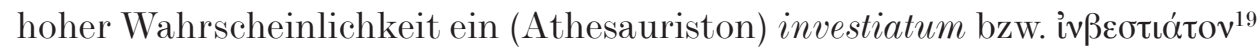
oder ein ähnliches Derivat von investitum $=$,,mit einem Amt bekleidet ${ }^{“ 20}$.

19 Und zwar unter der (graphischen) Voraussetzung, daß für einen mit lateinischen Buchstaben eher nur mit Mühe zu Rande kommenden Griechen die Ähnlichkeit zwischen einem $G$ in Uncialis (vgl. die beiden obigen Wiedergaben des NIREGIATON und des AGENTES aus dem Lipsiensis) und einem griechischen Stigma (= „st“) sehr groß war.

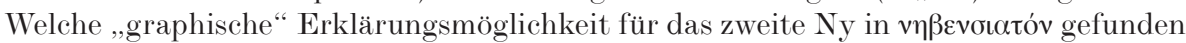
werden könnte, vermag ich hingegen nicht zu sagen. Grundsätzlich nicht (zumindest nicht a limine) auszuschließen wäre eine (irrtümliche) (griechische) Nasalierung vor

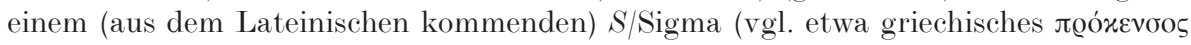
aus lateinischem processus; weitere Belege bei St. B. Psaltes, Grammatik der byzantinischen Chroniken [Forschungen zur griechischen und lateinischen Grammatik

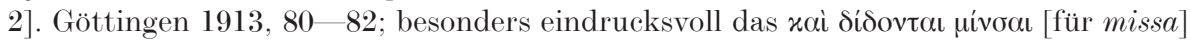
in De cer. I $40=$ Reiske I 205, $2-3$ [Lips., f. 93 ${ }^{\mathrm{v}}$, Z. 16], doch ergäbe eine derartige

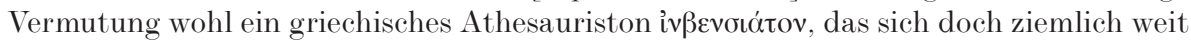
von der mit ziemlicher Sicherheit anzusetzenden lateinischen Wurzel invest- entfernt hätte).

${ }^{20}$ Analoge Ableitungen von investire - etwa investitio, investitor, investitura oder investitus - sind im späten oder mittelalterlichen Latein ausreichend belegt; vgl. etwa die Zusammenstellung bei J. F. Niermeyer-C. van de Kieft-J. W. J. Burgers, Mediae latinitatis lexicon minus. Édition remaniée, Bd. I. Leiden (Darmstadt) 2002, 728 - 730.

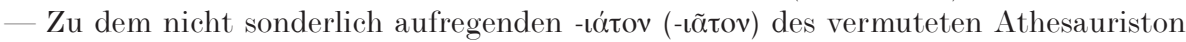

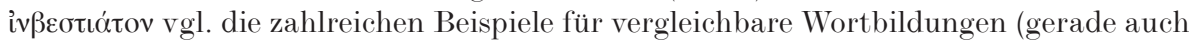
aus der „offiziellen Sprache“ der Titel und Ämter) bei N. B. Tomadakes, Tà cỉs -atum>

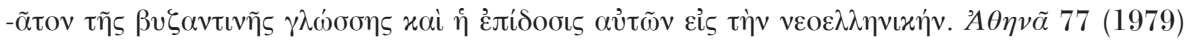

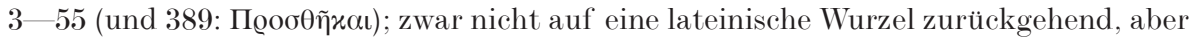

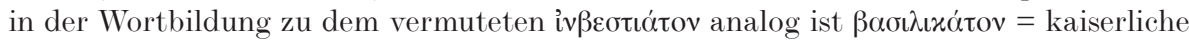
Schenkung (vgl. LBG I 268 s. v.). 